\title{
Analysis of depth-sensing indentation tests with a Knoop indenter
}

\author{
L. Riester \\ Oak Ridge National Laboratory, MS 6069, P.O. Box 2008, Oak Ridge, Tennessee 37831
}

\author{
T.J. Bell and A.C. Fischer-Cripps ${ }^{\text {a) }}$ \\ CSIRO Division of Telecommunications and Industrial Physics, P.O. Box 218, \\ Lindfield, NSW 2070, Australia
}

(Received 6 September 2000; accepted 21 March 2001)

\begin{abstract}
The present work shows how data obtained in a depth-sensing indentation test using a Knoop indenter may be analyzed to provide elastic modulus and hardness of the specimen material. The method takes into account the elastic recovery along the direction of the short axis of the residual impression as the indenter is removed. If elastic recovery is not accounted for, the elastic modulus and hardness are overestimated by an amount that depends on the ratio of $E / H$ of the specimen material. The new method of analysis expresses the elastic recovery of the short diagonal of the residual impression into an equivalent face angle for one side of the Knoop indenter. Conventional methods of analysis using this corrected angle provide results for modulus and hardness that are consistent with those obtained with other types of indenters.
\end{abstract}

\section{INTRODUCTION}

Indentation testing on the submicron scale enables convenient measurement of the mechanical properties of thin films and very small volumes of materials. Usually, the principal goal of such testing is to extract elastic modulus and hardness of the specimen from experimental readings of indenter load and depth of penetration. The methods of determining the area of contact from depth measurements, and hence hardness, and extraction of modulus from the unloading response are founded upon the elastic equations of contact of Hertz and also Sneddon. ${ }^{1-6}$ The methods rely on the analysis of the shape of the elastic unloading curve following elastic plastic contact between an indenter and a specimen. The most common indenter geometries used are the threesided Berkovich indenter, the four-sided Vickers indenter, and the spherical indenter. These indenters have some degree of geometrical symmetry about them that is not shared by the less commonly used Knoop indenter. The Knoop indenter has an elongated four-sided pyramidal geometry that has the advantage of providing very shallow depths of penetration and the ability to respond to differences in the ratio of hardness to modulus of the specimen material. This response manifests itself in the relative sizes of the lengths of the diagonals of the

\footnotetext{
a) Address all correspondence to this author.
}

residual impression. In the present work, we show how the more commonly used methods of analysis (e.g., multiple-point unload ${ }^{4}$ and single-point unload methods $^{5}$ ) may be modified to apply to load and depth data obtained with a Knoop indenter. The new method of analysis takes into consideration the elastic recovery of the specimen material during unloading which is assumed to be a function of $E / H$ of the specimen material. Comparison with experimental data is presented to illustrate the theory.

\section{ANALYSIS OF LOAD-DISPLACEMENT DATA}

\section{A. Berkovich indenter}

The three-sided Berkovich indenter has an included face angle of $\theta=65.3^{\circ}$, giving a projected area $A$ of the indentation as a function of the depth $h_{\mathrm{p}}$ beneath the contact as

$$
\begin{aligned}
A & =3 \sqrt{3} h_{\mathrm{p}}{ }^{2} \tan ^{2} 65.3, \\
& =24.5 h_{\mathrm{p}}{ }^{2} .
\end{aligned}
$$

It is convenient to regard the pyramidal geometry of a Berkovich indenter as an axis-symmetric cone for the purposes of analysis. The equivalent cone semiangle $\alpha_{\mathrm{i}}$ is calculated from

$$
\tan \alpha_{i}=\left(\frac{3 \sqrt{3} \tan ^{2} 65.3}{\pi}\right)^{1 / 2} .
$$


Upon unloading, the contact response is elastic and the relationship between the load and the depth of penetration for a cone is given by ${ }^{6}$

$$
P=\frac{2 E^{*}}{\pi} h_{\mathrm{e}}^{2} \tan \alpha^{\prime},
$$

where $\alpha^{\prime}$ is now the combined angle of the indenter and the residual impression, $E^{*}$ is the combined modulus of the specimen and the indenter, ${ }^{4}$ and $h_{\mathrm{e}}$ is the difference in the depth of penetration at full load and the depth of the residual impression at full unload. The normal displacement $h$ of points on the surface beneath the indenter is a function of the radial distance $r$ from the axis of symmetry and is given by

$$
h=\left(\frac{\pi}{2}-\frac{r}{a}\right) a \cot \alpha^{\prime} \quad r \leqslant a .
$$

As shown in Figure 1, as the indenter is unloaded, then the tip of the indenter (at $r=0$ ) moves through a distance $h_{\mathrm{e}}$ and the edge of the circle of contact with the specimen surface (at $r=a$ ) moves through a distance $h_{\mathrm{a}}$. Making use of Eq. (4), at load $P_{\mathrm{t}}$ the displacements $h_{\mathrm{e}}$ and $h_{\mathrm{a}}$ are thus

$$
\begin{aligned}
& h_{\mathrm{e}}=\frac{\pi}{2} a \cot \alpha^{\prime}, \\
& h_{\mathrm{a}}=\left(\frac{\pi}{2}-1\right) a \cot \alpha^{\prime},
\end{aligned}
$$

and hence

$$
h_{\mathrm{a}}=\left(\frac{\pi-2}{\pi}\right) h_{\mathrm{e}} .
$$

Now, from Fig. 1(b), we have

$$
\begin{aligned}
h_{\mathrm{t}} & =h_{\mathrm{p}}+h_{\mathrm{a}} \\
& =h_{\mathrm{p}}+\frac{\pi-2}{\pi} h_{\mathrm{e}} .
\end{aligned}
$$

The multiple-point unload method uses the slope of the tangent to the initial unloading to determine the quantities of interest. From Eq. (3), the slope of the elastic unloading is given by

$$
\frac{\mathrm{d} P}{\mathrm{~d} h}=2 \frac{2 E^{*} \tan \alpha^{\prime}}{\pi} h_{\mathrm{e}}
$$

Substituting back into Eq. (3), we have

$$
P=1 / 2 \frac{\mathrm{d} P}{\mathrm{~d} h} h_{\mathrm{e}} .
$$

Substituting Eq. (10) into Eq. (6) and letting $P=P_{\max }$, we have

$$
h_{\mathrm{a}}=\left[\frac{2(\pi-2)}{\pi}\right] \frac{P_{\max }}{\mathrm{d} P / \mathrm{d} h} .
$$

$h_{\mathrm{p}}$ can now be found from Eq. (7), which leads to the projected area of contact $A$ and hence hardness $H$. The bracketed term in Eq. (11) is termed an "intercept
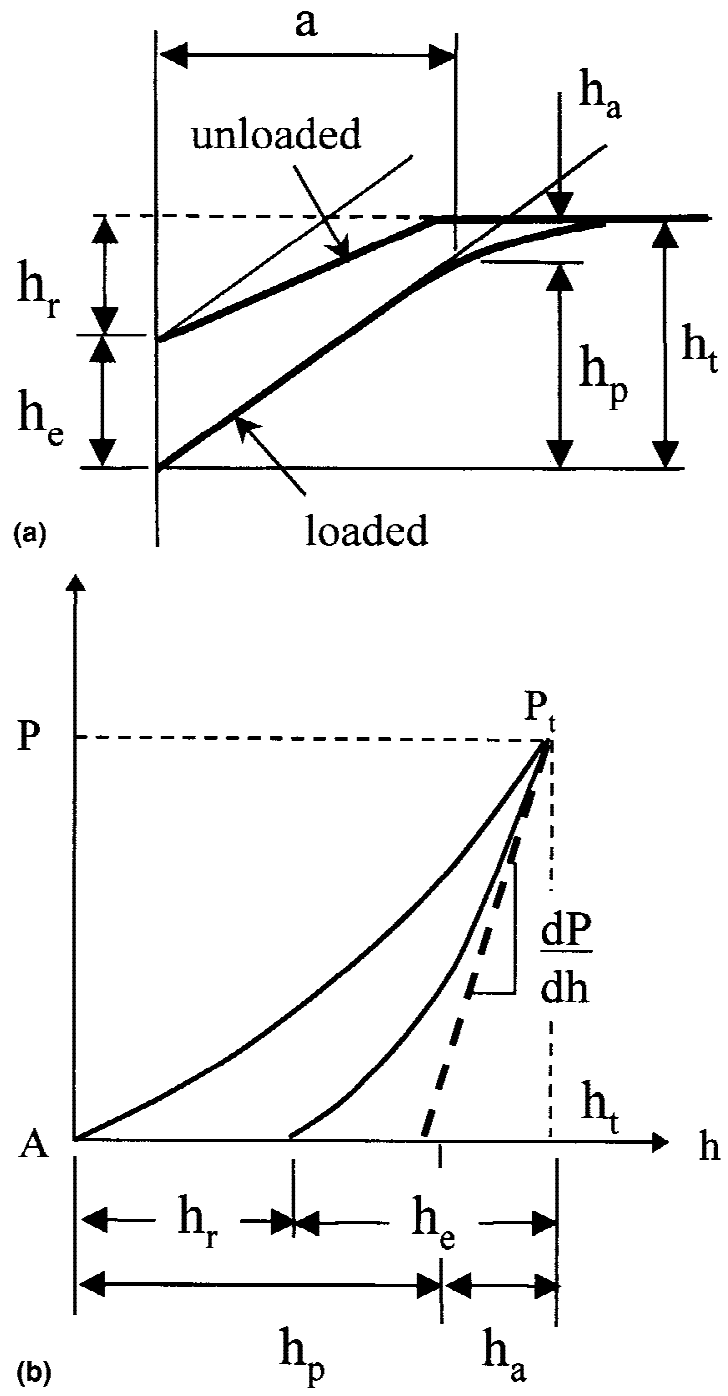

FIG. 1. (a) Schematic diagram of indenter and specimen surface geometry at full load and full unload for conical indenter. (b) Load versus displacement for elastic-plastic loading followed by elastic unloading. $h_{\mathrm{r}}$ is the depth of the residual impression, $h_{\mathrm{t}}$ is the depth from the original specimen surface at maximum load $P_{\mathrm{t}}, h_{\mathrm{e}}$ is the elastic displacement during unloading, and $h_{\mathrm{a}}$ is the distance from the edge of the contact to the specimen surface at full load. Upon elastic reloading, the tip of the indenter moves through a distance $h_{\mathrm{e}}$, and the eventual point of contact with the specimen surface moves through a distance $h_{\mathrm{a}}$.

correction factor" which evaluates to 0.72 for a cone as shown above, or exactly 0.75 for a sphere and unity for a cylindrical punch. Oliver and Pharr ${ }^{4}$ find that a value of 0.75 should be used as a result of the inevitable rounding of the tip of real pyramidal indenters. The combined modulus of the system can be determined from the slope of the initial unloading: ${ }^{7}$

$$
E^{*}=\frac{\mathrm{d} P}{\mathrm{~d} h} \frac{\sqrt{\pi}}{2 \beta} \frac{1}{\sqrt{A}},
$$

where $A$ can be found from Eq. (1), and $\beta=1.034$ is a geometry correction term to be applied for a Berkovich indenter ${ }^{8}$ and accounts for the nonaxissymmetric nature 
of the triangular pyramid geometry. The above analysis ignores any materials-related effects such as piling up and sinking in.

\section{B. Knoop indenter}

The analysis method described above for the Berkovich indenter relies on the conversion of the actual indenter geometry to an equivalent cone. That is, the elastic theory is applied to the unloading for a conical indenter of semiangle $\alpha$ from a preformed impression in the specimen surface. Various adjustments may be made to account for real indenter geometry. A similar analysis may be applied to the case of Vickers, comer cube, and other indenters. However, an interesting issue arises for the case of a Knoop indenter. A Knoop indenter is a foursided pyramidal indenter with unequal angles such as shown in Fig. 2 and where the projected area of contact is given by

$$
A=\frac{d^{2}}{2}\left[\cot \theta_{1} \tan \theta_{2}\right],
$$

where $\theta_{1}=86.25^{\circ}$ and $\theta_{2}=65^{\circ}$ and $d$ is the length of the long diagonal of the residual impression. Expressed in terms of the plastic depth $h_{\mathrm{p}}$, Eq. (13) becomes

$$
A=2 h_{\mathrm{p}}{ }^{2} \tan \theta_{1} \tan \theta_{2} \text {. }
$$

As will be shown below, analysis of experimental data obtained with a Knoop indenter on fused silica, using the methods above for an equivalent cone angle of $77.64^{\circ}$, show that both the hardness and the modulus are overestimated. The reason is that, in this material, there is substantial elastic recovery of the short diagonal of the residual impression compared with negligible elastic recovery of the long axis direction. The long axis of the impression made by a Knoop indenter is approximately seven times larger than the short axis at full load. Upon removal of load, elastic strains stored within the material are relaxed as the specimen material attempts to regain its original shape. Now, since the long axis of the impression made by a Knoop indenter is much greater than the short axis, the restoring forces perpendicular to the long axis (i.e., those resulting from the relaxation of elastic strains on the short axis) have a much longer "moment arm" than those perpendicular to the short axis. In other words, the sides "collapse" inward as the indenter is withdrawn. (A similar effect is demonstrated when breaking an egg by pressing along the long axis as compared with along the short axis. ${ }^{9}$ ) This means that observed elastic recovery in the short axis direction can be substantial compared to that in the long axis direction, especially for materials with a low value of $E / H$ where elastic recovery is more pronounced. Other indenters (such as Vickers and Berkovich) while not axissymmetric have equal lengths of axes, and there is an equal balance of restoring forces on the specimen material during unloading.

The observed differences in elastic recovery on the axes of a Knoop indenter for highly elastic materials have been widely reported in the literature, and there exists a small number of theoretical treatments to account for this behavior. Marshall, Noma, and Evans ${ }^{10}$ likened the elastic recovery for a Knoop indenter to that of an elliptical cone with major and minor axes and applied elasticity theory to arrive at an expression for the recovered indentation size in terms of the geometry of the indenter and the ratio $E / H$ (see Fig. 2).

$$
\frac{b^{\prime}}{d^{\prime}}=\frac{b}{d}-\alpha \frac{H}{E}
$$

In Eq. (15), $\alpha$ is a geometry factor found from experiments on a wide range of materials ${ }^{10}$ to be equal to 0.45 . The ratio of the dimension of the short diagonal $b$ to the long diagonal $d$ at full load is given by the indenter geometry, and for a Knoop indenter, $b / d=1 / 7.11$. The primed values of $d$ and $b$ are the lengths of the long and short diagonals after removal of load. Since there is observed to be negligible recovery along the long diagonal, we can say that $d^{\prime} \approx d$. When $H$ is small and $E$ is large (e.g., metals), then $b^{\prime} \approx b$ indicating negligible elastic recovery along the short diagonal. When $H$ is large and $E$ is small (e.g., glasses and ceramics), there we would expect $b^{\prime} \ll b$.

The elastic analysis described previously for the Berkovich indenter relies on Sneddon's solution for a conical indenter in which the depth as a function of load is given

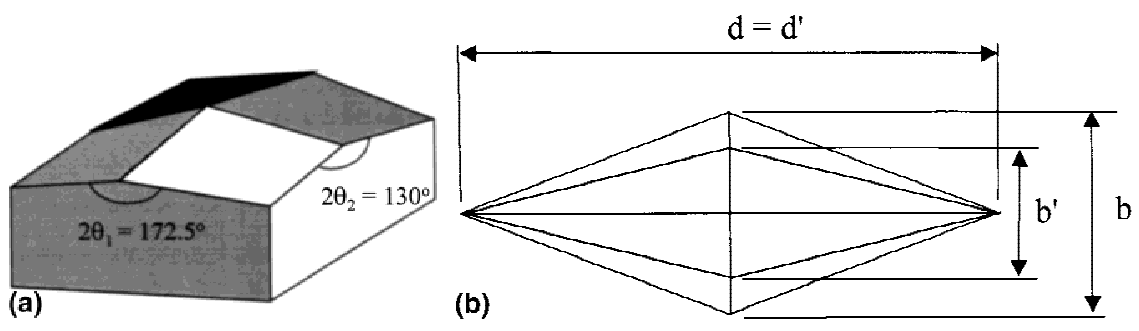

FIG. 2. (a) Geometry of Knoop indenter. (b) At full load, the length of the long diagonal at the contact depth is 7.11 times as long as the length of the short diagonal. After unloading, elastic recovery along the direction of the short axis diagonal means that the distance $b$ reduces to $b^{\prime}$. Very little elastic recovery is seen along the direction of the long axis so that $a=a^{\prime}$. 
by Eq. (3). For materials with a low value of $E / H$ (e.g., metals) we would expect these analysis methods to give acceptable results even for a Knoop indenter since the amount of elastic recovery is small. For glass and ceramics, the experimental readings of load and displacement for a Knoop indenter will be affected by the elastic recovery along the short axis dimension and this will not be accommodated by Eq. (3). Upon loading, to reach a particular depth of penetration, we would need to apply a higher value of load compared with an equivalent conical indenter to overcome the elastic recovery forces arising from the elastic recovery along the short axis direction. Thus, in an experiment involving a Knoop indenter on, for example, glass, the depth of penetration at any particular load would be less than for an equivalent conical indenter.

The degree of elastic recovery expressed in terms of the length of the short diagonal of the residual impression is quantified empirically by Eq. (15). Figure 3 shows the region of interest about the short axis of a Knoop indenter. For a load/unload cycle with a Knoop indenter, elastic recovery forces act in addition to those experienced by an equivalent cone due to the required compression, and subsequent expansion, of the "elastic recovery volume" $\mathrm{ABC}$ in Fig. 3. This volume goes to zero as the dimension $b^{\prime}$ approaches $b$. It is possible to account for this in the analyses methods given here by increasing the effective angle $\theta_{2}$ so that compression and relaxation of the elastic recovery volume is accommodated. How much should the angle $\theta_{2}$ be adjusted? Evidently, the adjustment should be a function of $E / H$ and

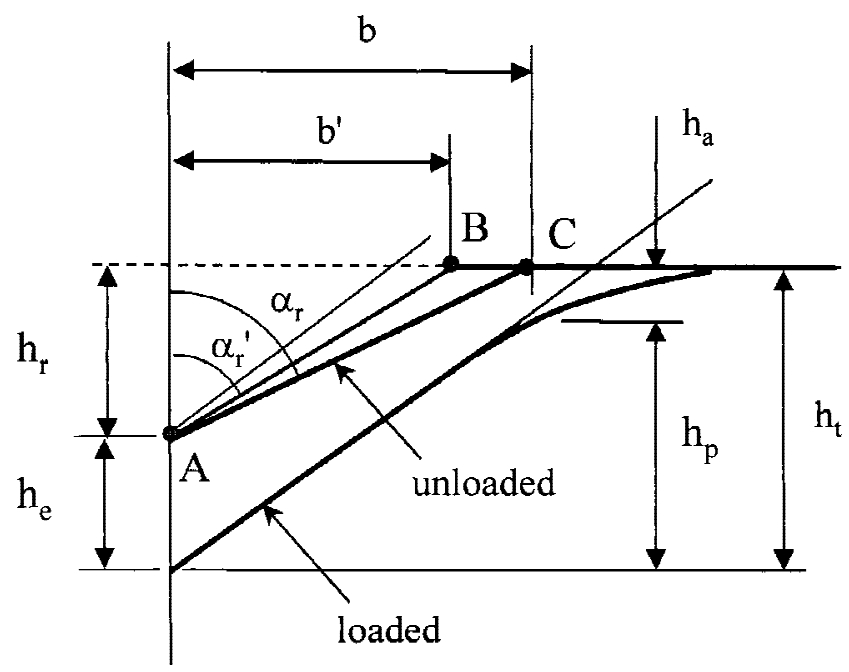

FIG. 3. Schematic diagram of the geometry of contact near the recovered impression made by Knoop indenter. When there is elastic recovery along the short axis of the diagonal, the dimension of the short diagonal changes from $b$ to $b^{\prime}$ and the corresponding change in the angle of the residual impression is from $\alpha_{\mathrm{r}}$ to $\alpha_{\mathrm{r}}{ }^{\prime}$. incorporate the results of Eq. (15). Rearranging Eq. (15) assuming that there is no recovery along the long axis such that $d^{\prime}=d$, then

$$
\frac{b^{\prime}}{b}=1-\alpha \frac{d}{b} \frac{H}{E}
$$

where $d / b=7.11$ and $\alpha=0.45 .{ }^{10}$ With reference to Fig. 3, it can be seen that the angle of the residual impression changes from $\alpha_{\tau}$ to $\alpha_{\tau}{ }^{\prime}$ according to

$$
\frac{\tan \alpha_{\mathrm{r}}{ }^{\prime}}{\tan \alpha_{\mathrm{r}}}=\frac{b^{\prime}}{b} \text {. }
$$

Let us assume that the same fractional change of angle may be attributed to the proposed increase in angle $\theta_{2}$ of the indenter. The corrected angle $\theta_{2}{ }^{\prime}$ for the Knoop indenter which accounts for elastic recovery forces is thus

$$
\tan \theta_{2}{ }^{\prime}=\left(\frac{b^{\prime}}{b} \tan \theta_{2}\right),
$$

where $b / b^{\prime}$ is given by Eq. (16). For the purposes of analyses, we can immediately see that an initial guess at the ratio of $E / H$ is required for insertion into Eq. (16). $E$ and $H$ can then be calculated by the methods described above and the ratio $E / H$ adjusted for convergence. It should be noted that absolute values for $E$ and $H$ are not required initially but only their ratio. Thus, an informed initial estimate should result in a fairly rapid convergence.

\section{COMPARISON WITH EXPERIMENTAL DATA AND DISCUSSION}

It is of interest to evaluate the applicability of the analysis by comparison with nominal values of modulus and hardness with those calculated from the experimental data. Experiments for this purpose were conducted using a UMIS ${ }^{11}$ indentation instrument with a Knoop indenter on fused silica, a standardized steel hardness block, and a sample of alumina. The nominal values of modulus and hardness for these materials are 70 and $7.6 \mathrm{GPa}^{12}$ 203 and $8.3 \mathrm{GPa},{ }^{13}$ and 380 and $18 \mathrm{GPa},{ }^{14}$ respectively, representing a range of hardness and modulus combinations. It is well-known that the indentation response of materials is dependent on the ratio of $E / H$, where $H$ is the hardness related to the yield stress through a constraint factor. The materials studied here represent a wide range of $E / H$ being approximately 9 for fused silica, 24 for steel, and 21 for alumina. For each material studied, a relatively large maximum load $(500 \mathrm{mN})$ was used so that the elastic recovery factor of the residual impressions could be measured optically and compared with those calculated from the load displacement data. A constant load rate was used throughout, and the results presented here were the average of four indentations on each specimen. The standard error for maximum depths of penetration was $0.11 \%$ for fused silica, $0.6 \%$ for the steel, and $0.5 \%$ for the alumina specimens. 
Elastic modulus and hardness were calculated using the analysis procedures described above. Multiple unload points from maximum load were taken, and the multiple-point unload method was used to determine elastic modulus and hardness. In all cases, data from the upper $10 \%$ of the unloading were used for the calculations. A Poisson's ratio of 0.22 was assumed for the fused silica and the alumina, and 0.3 for the hardened steel in the analyses. Values of $E$ and Poisson's ratio used for the diamond indenters were $1000 \mathrm{GPa}$ and 0.07 , respectively. Nominal values for modulus and hardness for each material were used for determining the ratio $E / H$ for the analysis and also the results from a simulation calculation of load displacement curves, and these are shown in Table I. In all analyses, an intercept factor of 0.75 was used. A geometry correction factor of $\beta=1.034$ was used for the Knoop analyses with $\beta=1.0$ for the cone analyses. ${ }^{4}$ It could be argued that $\beta=1.012$, that found by $\mathrm{King}^{8}$ to be applicable for a Vickers indenter, might be more appropriate for the Knoop indenter, but the final results do not significantly depend on the actual value chosen. The results were

TABLE I. Summary of analysis results for three specimen materials. For each material, columns show results of analysis of experimental data taken with a Knoop indenter using the new method of analysis that corrects for elastic recovery and results of analysis of experimental data using the assumption of an equivalent cone angle of $77.64^{\circ}$ and no correction for elastic recovery. Data presented are the following: elastic modulus; hardness from analysis of unloading; maximum penetration depth $h_{\mathrm{t}}$; residual depth $h_{\mathrm{r}}$; elastic recovery factor calculated from nominal values of $E$ and $H$, ERF; elastic recovery factor calculated from measurements of experimental residual impressions (observed); the equivalent cone angle based upon the dimensions of the Knoop indenter and taking into consideration elastic recovery. The \% difference between the calculated values and the nominal values of $E$ and $H$ are shown. For maximum penetration depth and depth of residual impression, the results of the simulation calculation based upon the nominal values of modulus and hardness are given. The residual depth estimated from the intercept of the experimental loadpenetration curve with the depth axis is also shown ( $h_{\mathrm{r}}$ observed).

\begin{tabular}{|c|c|c|c|c|c|c|}
\hline \multirow[b]{3}{*}{ Parameter } & \multicolumn{6}{|c|}{ Analysis type } \\
\hline & \multicolumn{2}{|c|}{ Steel } & \multicolumn{2}{|c|}{ Fused silica } & \multicolumn{2}{|c|}{ Alumina } \\
\hline & Knoop & Cone & Knoop & Cone & Knoop & Cone \\
\hline$E(\mathrm{GPa})$ & 213 & 241 & 67.8 & 86.6 & 394 & 457 \\
\hline$\%$ diff & $4.9 \%$ & $19 \%$ & $-3 \%$ & $24 \%$ & $3.7 \%$ & $20 \%$ \\
\hline$H(\mathrm{GPa})$ & 8.5 & 9.7 & 5.5 & 8.1 & 16.0 & 18.4 \\
\hline$\%$ diff & $2.8 \%$ & $16.7 \%$ & $-27 \%^{\mathrm{a}}$ & $6.6 \%$ & $-11 \%$ & $2.1 \%$ \\
\hline$h_{\mathrm{t}}(\mu \mathrm{m})$ & 1.05 & 1.05 & 1.41 & 1.41 & 0.77 & 0.77 \\
\hline Simulated & 1.09 & & 1.40 & & 0.81 & \\
\hline$h_{\mathrm{r}}(\mu \mathrm{m})$ & 0.46 & 0.46 & 0.056 & 0.056 & 0.25 & 0.25 \\
\hline Simulated & 0.47 & & 0 & & 0.25 & \\
\hline Obsd. & 0.60 & & 0.45 & & 0.34 & \\
\hline ERF & $86.3 \%$ & 0 & $65.3 \%$ & 0 & $84.4 \%$ & 0 \\
\hline Obsd. & $97.5 \%$ & & $70.0 \%$ & & $85.3 \%$ & \\
\hline$\alpha(\operatorname{deg})$ & 78.5 & 77.6 & 80.0 & 77.6 & 78.6 & 77.6 \\
\hline
\end{tabular}

${ }^{a}$ Underdeveloped plastic zone. adjusted for the shape function of the indenter. This was established by performing a series of tests on the fused silica sample at a range of maximum loads and then using the analysis method in reverse together with the nominal value of elastic modulus and correcting for elastic recovery to arrive at an area function which related the actual area of contact $A$ with the ideal area of contact $A_{\mathrm{i}}$. The area function so obtained is shown as the ratio $A / A_{\mathrm{i}}$ against the plastic depth $h_{\mathrm{p}}$ in Fig. 4 . The results for load and displacement for all experiments were also corrected for instrument compliance (estimated to be $1 \times 10^{7} \mathrm{~N} / \mathrm{m}$ ) and initial penetration depth.

Figures 5-7 show the load-displacement responses from the experiments and also from a simulation calculation ${ }^{15}$ using the nominal values of elastic modulus and hardness as inputs. Table I shows the results obtained for each specimen. The first column for each specimen material shows results from experimental data corrected for elastic recovery, and the second column shows the values obtained from experimental data using the uncorrected analysis where the Knoop indenter was considered as a cone with an equivalent angle of $77.64^{\circ}$. The "elastic recovery factor" ("ERF" in Table I) is the length of the recovered short diagonal $b^{\prime}$ expressed as a percentage of the unrecovered length $b$ calculated from the nominal values of $E$ and $H$ for each specimen. Also shown is the

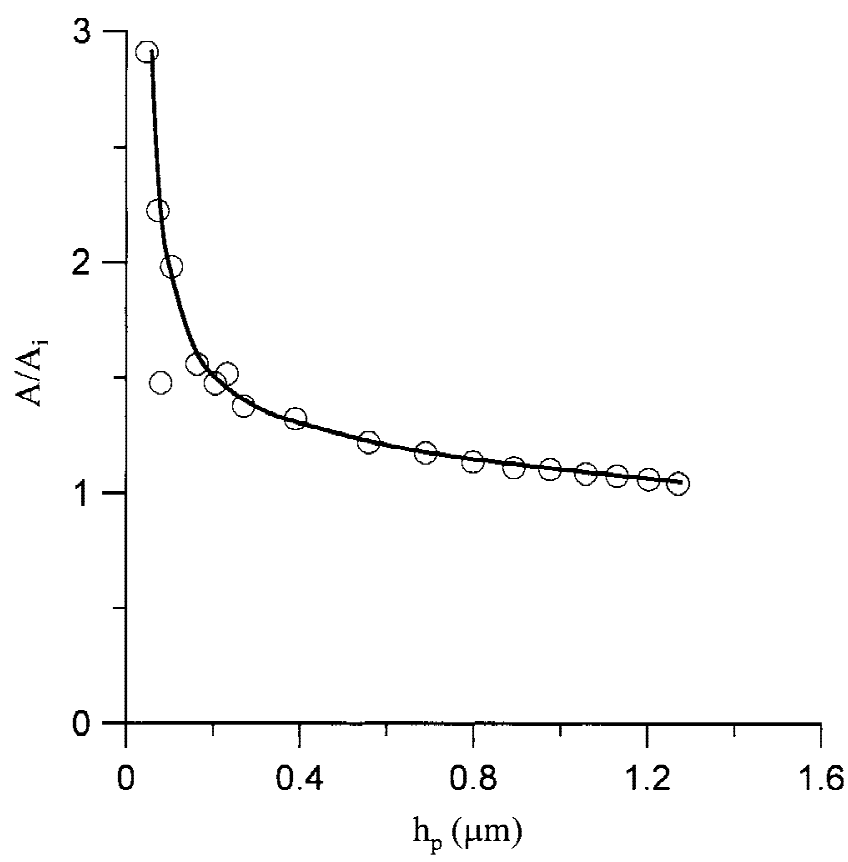

FIG. 4. Area function of Knoop indenter as found on indentation tests on fused silica with $E=70 \mathrm{GPa}$ and $H=7.6 \mathrm{GPa}$ with $v=0.22$. The vertical axis shows the ratio of the actual area of contact $A$ divided by the ideal area of contact $A_{\mathrm{i}}$ for an indenter with perfect geometry at any given value of the plastic depth $h_{\mathrm{p}}$. A ratio of $A / A_{\mathrm{i}}=1$ indicates that the actual shape of the indenter is identical to the expected shape. Open circles indicate raw data, and the solid line is a smooth curve fitted to data and used to calculate the indenter shape correction factor $A / A_{\mathrm{i}}$ for a given value of $h_{\mathrm{p}}$. 
elastic recovery factor calculated from measurements on the residual impressions in the specimen surfaces as shown in Figs. 5-7. Finally, an equivalent cone angle is given which enables the relative "bluntness" of the various indenter geometries to be compared.

It is immediately evident from the data in Table I that, for the hardened steel specimen, the values of modulus and hardness obtained without correcting for elastic

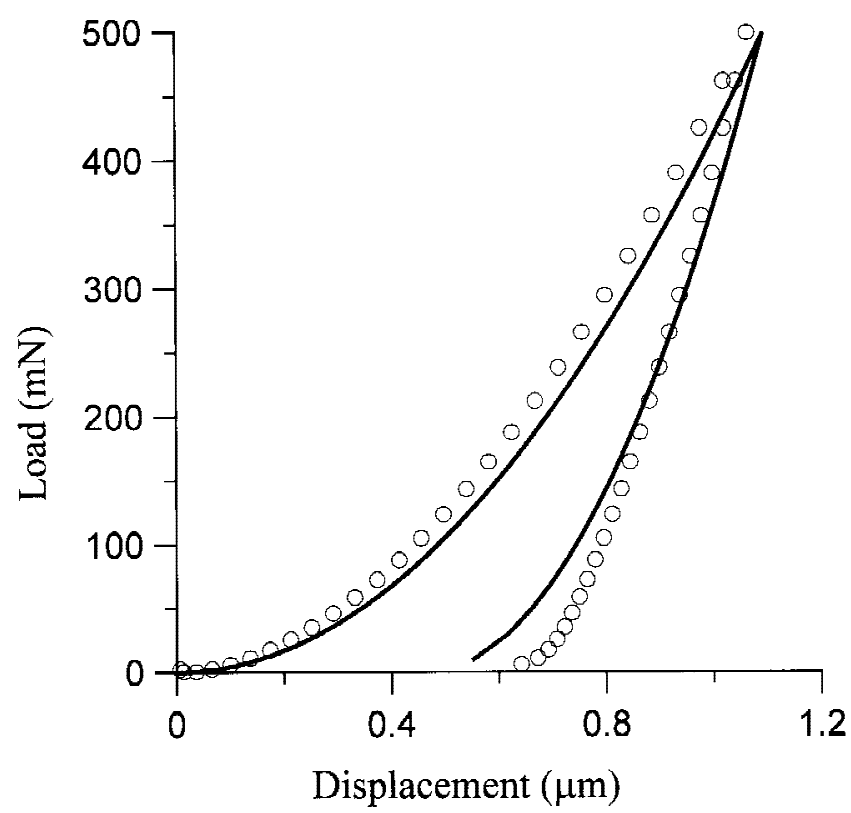

FIG. 5. Load-displacement curve for Knoop indenter on hardened steel. Data points indicate the experimental results, and the solid line represents simulation calculation using nominal values of $H$ and $E$.

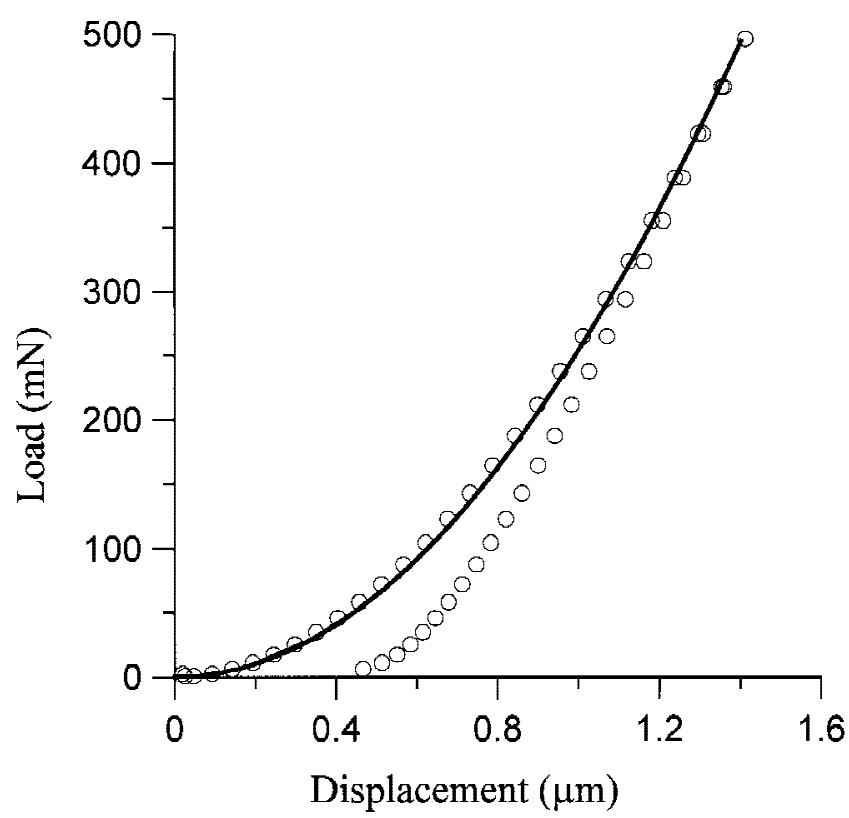

FIG. 6. Load-displacement curve for Knoop indenter on fused silica. Data points indicate experimental results, and the solid line represents simulation calculation using nominal values of $H$ and $E$. recovery effects (the "cone" analysis) are overestimated by $19 \%$ and $17 \%$, respectively, compared to nominal values. Correcting for elastic recovery results in a $4.9 \%$ increase in the estimation of modulus and a $2.8 \%$ increase in hardness compared to nominal values. For this material, the elastic recovery effect is relatively small and is quantified by the calculated value of "ERF" in the table at $86.3 \%$ (i.e., $\mathrm{ERF}=100 \%$ indicating no elastic recovery of the short diagonal). The values of maximum penetration and residual depth calculated using the simulation calculation are consistent with those estimated from an analysis of the slope of the experimental unloading curve.

For the fused silica sample, Table I shows that the new procedure underestimates the modulus by about $3 \%$ while the uncorrected procedure overestimates the modulus by $24 \%$. The new procedure underestimates the hardness value by approximately $27 \%$ while the uncorrected procedure overestimates the hardness by about $7 \%$. The reason for the seemingly poor correlation with hardness is that, for a conical indenter, the mean contact pressure depends upon the cone semiangle and the modulus of the specimen material (and independent of the load due to geometrical similarity) according to

$$
p_{\mathrm{m}}=1 / 2 \frac{E}{\left(1-v^{2}\right)} \cot \alpha .
$$

Now, the Knoop indenter is very "blunt" and made more so by the correction to the angle $\theta_{2}$ arising from elastic recovery as described in the present paper. If for

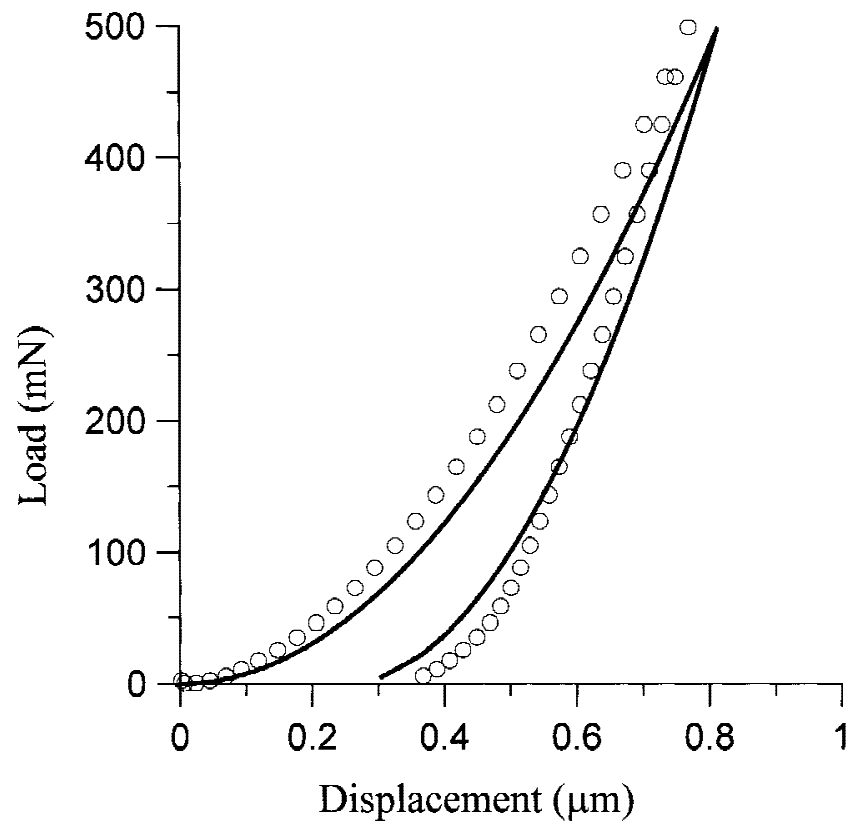

FIG. 7. Load-displacement curve for Knoop indenter on alumina. Data points indicate experimental results, and the solid line represents simulation calculation using nominal values of $H$ and $E$. 
fused silica, we take the hardness as being 7.6 GPa (with $E=70 \mathrm{GPa}$ and $v=0.22$ ) and inserting this as the mean contact pressure in Eq. (19), we find that the limiting angle for a plastic impression is $\alpha=78.3^{\circ}$. Any conical indenter with an included half-angle greater than this will result in a mean contact pressure less than the nominal hardness of the material. Now, this is normally not an issue with Vickers or Berkovich indenters since the equivalent cone angles are much smaller than this. However, in the present work, the Knoop indenter results in an equivalent cone angle of $80^{\circ}$ (see Table I), which means that, theoretically, no matter what the load, the mean contact pressure is less than the hardness of the material. The elastic analysis (both the analysis and the simulation) depend on the mean contact pressure being equal to the hardness of the material. The multiplepoint unload method of analysis (the "Oliver and Pharr" method) uses the tangent to the slope of the elastic unloading curve which is assumed to follow that of Sneddon's analysis for the unloading of a cone. It is a straightforward matter to determine the expected shape of the unloading curve and extrapolate this back to zero load to determine an expected value of the depth of the residual impression. When this is done for the fused silica results, the expected depth of the residual impression is $0.056 \mu \mathrm{m}$; that is, the analysis predicts an almost elastic response in accordance with the simulation calculation. However, the experimental evidence indicates that there is indeed a substantial depth of residual impression. Physically, one would expect some plastic deformation in the specimen due to the singularity of stresses at the tip of the indenter, and this is indicated in the experimental data in Fig. 6. However, since the analysis methods used to determine hardness depend on elastic equations, it is presumed that in these analysis methods that the mean contact pressure computed by the elastic equations will be greater than the hardness of the material. Evidently, for very blunt indenters this cannot occur and, thus, the method of analysis breaks down. The computation of modulus is not affected, and hence, the determination of the area function from the experimental results remains valid. Thus, the reason for the low values of hardness predicted for the fused silica specimen in Table I is that the mean contact pressure computed by the elastic equations is less than that of the actual hardness of the material. The reason that this same problem does not occur for the alumina sample (which is harder than fused silica) is that the mean contact pressure depends on both the cone angle and the specimen modulus. The modulus of the alumina is very much higher than that of fused silica, and so for the same cone angle, the mean contact pressure is consequently higher since the contact area is reduced. Thus, we do expect a fully developed plastic zone for the alumina but not for the fused silica specimen.
It should be remembered that we used a series of indentations on the fused silica sample to establish an area correction function for the indenter shape as shown in Fig. 4. We should therefore not be surprised to see a close comparison in elastic modulus for the new procedure and the nominal value of modulus. However, the validity of the new procedure is tested, somewhat indirectly, by using the same indenter area function in the analysis for the other two materials, with different ratios of $E / H$ considered in the present work, and we comment upon this further below.

For the alumina sample, Table I shows that the new method of analysis overestimates the modulus by about $3.7 \%$, and the uncorrected analysis overestimates the modulus by $20 \%$. The new method of analysis underestimates the hardness by about $11 \%$, and the uncorrected analysis overestimates the hardness by $2 \%$. Considering the variability in properties associated with any ceramic material with processing schedule, we should not perhaps expect such a good correlation between the experimental readings and the nominal values as we have obtained for fused silica and the steel hardness block. Unlike the case of fused silica, there is a reasonable agreement between the simulated and experimental unloading curves and the depths of residual impression. This is a consequence of the combination of elastic modulus and effective cone angle resulting in a calculated mean contact pressure that is larger than the hardness of the material.

It is of interest to compare the calculated elastic recovery factor to that observed from measurements of the diagonals of the impressions in the experimental samples. Measurements taken from the micographs shown in Fig. 8 were used to calculate the observed elastic recovery factor, and these are compared with the calculated values in Table I. It can be seen that Eq. (16) provides a reasonable estimate of the elastic recovery factor if the ratio of $E$ and $H$ for the specimen is known beforehand. For the specimens shown in Fig. 8, the measured length of the long diagonals for a load of $500 \mathrm{mN}$ are $31 \mu \mathrm{m}$ for steel and 33 and $19.5 \mu \mathrm{m}$ for alumina. The calculated Knoop hardness values from the load divided by the area given by Eq. (3) are $7.4 \mathrm{GPa}$ for steel, 6.5 GPa for fused-silica, and 18.7 GPa for alumina. These values are consistent with the nominal values given previously except that the value of the measured hardness for the fused silica is somewhat lower than expected indicating a less than fully developed plastic zone for this material under the test conditions reported here.

The selection of the fused-silica specimen for determination of the area function of the indenter deserves comment. The indenter area function should cover a wide range of penetration depths so as to be applicable for results taken with a wide variety of specimen materials. The area function calibration is most important however at small values of penetration depth, i.e., near the tip of 


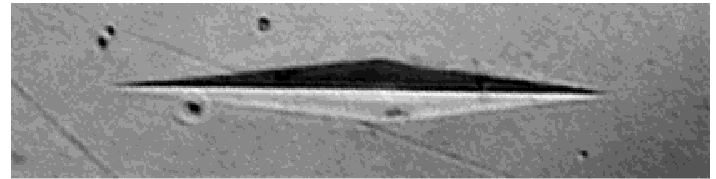

(a)

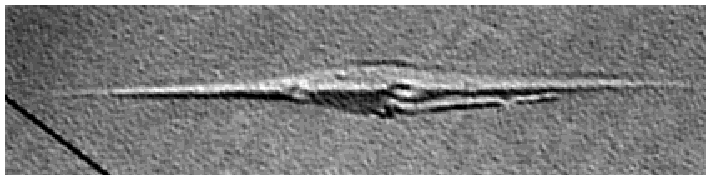

(b)

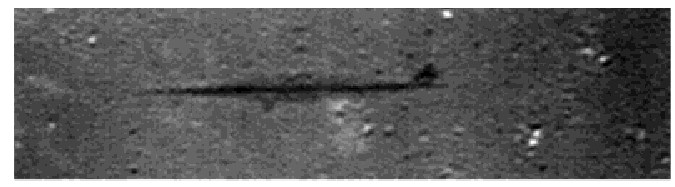

(c)

FIG. 8. Optical micrographs of a residual impression in specimen surface for a Knoop indenter at $500 \mathrm{mN}$ load for (a) hardened steel, (b) fused silica, and (c) alumina. The measured lengths of the long diagonals are (a) $31 \mu \mathrm{m}$, (b) $33 \mu \mathrm{m}$, and (c) $19.5 \mu \mathrm{m}$, and this provides a scale for the figure. The Knoop hardness values from Eq. (3) are (a) $7.4 \mathrm{GPa}$, (b) $6.5 \mathrm{GPa}$, and (c) $18.7 \mathrm{GPa}$.

the indenter where bluntness and damage are most likely. Fused silica is an excellent material for determining the indenter shape since it offers a reasonably high value of hardness and a low value of modulus thus permitting useful data to be generated over a wide range of penetration depths, unlike e.g., steel which would provide few data at low penetration depths and more data at larger penetration depths where the correction is not so important. No matter what material is selected for establishing an area function, it is important to note that the results depend upon the application of the new method of analysis presented here since this analysis takes into account the elastic recovery associated with the Knoop indenter. It should be further noted that the computed area function depends upon a known value of elastic modulus of the specimen material and not the hardness. While the procedure described here might appear to be somewhat circular, the overall soundness of the analysis is supported by the results obtained for the steel and the alumina specimens, not only for the computed values of $E$ and $H$ but also the correspondence between the experimental and simulated load-displacement curves shown in Figs. 6 and 8.

The analysis method for the Knoop indenter given in the present work incorporates the effect of the elastic recovery of the short axis diagonal by adjusting the corresponding face angle for the indenter to be large by approximately the same amount. This means that the extra volume to be compressed or released is accommodated. It should be remembered that this only applies for the Knoop indenter for which there is an unbalanced elastic recovery force due to the elongated geometry. In a Berkovich, Vickers, or conical indenter, elastic recovery of the specimen material is balanced uniformly and is therefore already incorporated into the Hertz equations.

\section{CONCLUSIONS}

The present work shows how conventional methods of analysis of depth-sensing indentation test data may be modified to suit the elastic recovery associated with indentations made with a Knoop indenter. Without this modification, both elastic modulus and hardness are overestimated by an amount that depends on the ratio of $E / H$ of the specimen material. The new method of analysis expresses the elastic recovery of the short diagonal of the residual impression into an equivalent face angle for one side of the Knoop indenter. Conventional methods of analysis using this corrected angle provide results for modulus and hardness that are consistent with those obtained with other types of indenters.

\section{ACKNOWLEDGMENT}

This research sponsored in part by the Assistant Secretary for Energy Efficiency and Renewable Energy, Office of Transportation Technologies, as part of the High Temperature Materials Laboratory User Program, Oak Ridge National Laboratory, managed by UT-Battelle, LLC, for the United States Department of Energy under Contract No. DE-AC05-000R22725.

\section{REFERENCES}

1. H. Hertz, J. Reine Angew. Math. 92, 156 (1881). Translated and reprinted in English in Hertz's Miscellaneous Papers (Macmillan \& Co., London, U.K., 1896), Chap. 5.

2. H. Hertz, Verh. Ver. Beförderung Gewerbe Fleisses 61, 410 (1881). Translated and reprinted in English in Hertz's Miscellaneous Papers (Macmillan \& Co., London, U.K., 1896), Chap. 6.

3. M.F. Doerner and W.D. Nix, J. Mater. Res. 1, 601 (1986).

4. W.C. Oliver and G.M. Pharr, J. Mater. Res. 7, 1564 (1992).

5. J.S. Field and M.V. Swain, J. Mater. Res. 8, 297 (1993).

6. I.N. Sneddon, Proc. Cambridge Philos. Soc. 44, 492 (1948).

7. G.M. Pharr, W.C. Oliver, and F.R. Brotzen, J. Mater. Res. 7, 613 (1992).

8. R.B. King, Int. J. Solids Struct. 23, 1657 (1987).

9. D.B. Marshall and B.R. Lawn, in Microindentation Techniques in Materials Science and Engineering, ASTM STP 889, edited by P.J. Blau and B.R. Lawn (American Society for Testing and Materials, Philadelphia, PA, 1986).

10. D.B. Marshall, T. Noma, and A.G. Evans, J. Am. Ceram. Soc. 65, C175 (1980).

11. CSIRO Division of Telecommunications and Industrial Physics, Lindfield, Australia.

12. I.J. McColm, Ceramic Hardness (Plenum Press, New York, 1990).

13. Yamamoto Scientific Tool Laboratory Co., Ltd., Chiba, Japan.

14. Rojan Advanced Ceramics Pty. Ltd., Spearwood, WA, Australia.

15. A.C. Fischer-Cripps, J. Mater. Res. (2000, accepted for publication). 